Suceveanu Andra-Iulia, Mazilu Laura, Voinea F., Suceveanu A.P., Parepa Irinel Raluca, Catrinoiu Doina, Tuta Liliana-Ana

\title{
Obesity, an independent risk factor for hepatocellular carcinoma [HCC] in NAFLD non-cirrhotic patients
}

Department of Internal Medicine, Faculty of medicine Constanţa, Romania

\begin{abstract}
Hepatocellular carcinoma (HCC) is one of the most common malignancies with increasing incidence in developed countries. Epidemiological studies show that the cause of new discovered HCC cases remains unclear in $15 \%-50 \%$ of cases. Obesity and the subsequent/ underlying nonalcoholic fatty liver disease (NAFLD) can be responsible for most of these cases. The aim of our study was to estimate the risk of HCC in obese patients diagnosed with NAFLD, without clinical or imagistic features of liver cirrhosis, in order to see if HCC can develop in fatty liver in the absence of cirrhosis. Patients with regular/daily alcohol consumption or diagnosed with liver viral infections were excluded. We studied 214 obese patients with NAFLD over a period of 5 years. We evaluated all patients using abdominal ultrasound and serum alpha-fetoprotein every 6 month, in order to detect the HCC occurrence. Kaplan-Meier analysis estimated the cumulative incidence of HCC. Univariate and multivariate Cox regression analysis were used to assess associations between HCC and obesity. The median follow-up was 4.3 years. During the study period, 16 from 118 cirrhotic NFLAD patients $(13.5 \%)$ and 12 from 96 non-cirrhotic NAFLD patients $(12.5 \%)$ developed HCC $(p=0.07, n s)$. The cumulative incidence of $\mathrm{HCC}$ was found to be $2.9 \%$ in obese patients with NAFLD-cirrhosis, compared with $2.2 \%$ in obese patients without cirrhosis $(p=0.09, n s)$. Multivariate regression analysis revealed that older age $(\mathrm{p}=0.04)$ was independent variable associated with
\end{abstract}

Suceveanu Andra-Iulia

Internal Medicine Department

Faculty of medicine, "Ovidius" University of Constanţa

Constanta County Emergency Hospital, 145 Tomis Blvd, Constanta andrasuceveanu@yahoo.com

Tel./Fax: +40-241-553022 development of HCC in patients with/without NAFLDcirrhosis. Obesity seems to be an independent risk factor for HCC occurrence, regardless the presence of mild or advanced liver fibrosis in NAFLD patients.

Keywords: obesity, hepatocellular carcinoma, NAFLD.

\section{Introduction}

Hepatocellular carcinoma (HCC) is one of the most common malignancies with increasing incidence in developed countries. In the setting of chronic hepatitis $\mathrm{B}(\mathrm{CHB})$ or $\mathrm{C}$ infection $(\mathrm{CHC})$, coexisting obesity has been shown to increase the risk for HCC by more than 100-fold [1]. Epidemiological studies show that the cause of new discovered HCC cases remains unclear in $15 \%-50 \%$ of cases $[2,3]$.Obesity and the underlying nonalcoholic fatty liver disease (NAFLD) can be responsible for most of these cases [4]. Liver cirrhosis is no longer a necessary condition for hepatocellular carcinoma occurrence. On the other, a large number of epidemiologic studies have shown that obesity increases the risk of a variety of cancers, including colon, renal, prostate, postmenopausal breast, and ovarian, in Asian and Western countries $[5,6,7]$. Obesity has recently received increased attention as risk factor for hepatocellular carcinoma $[8,9,10,11]$. Obesity has been shown to be involved in the progression of liver fibrosis; weather obesity is an independent risk factor for hepatocellular 
carcinoma (HCC) in NAFLD non-cirrhotic patients is a hypothesis to be demonstrated. In addition, obesity may impact disease progression, a large prospective trial revealing an association between increased weight and overall cancer mortality [12]. A body mass index $(\mathrm{BMI})>35 \mathrm{~kg} / \mathrm{m} 2$ negatively impact mortality from liver cancer with a 1.68 times relative risk (RR) in women and a 4.52 times $R R$ in men. Similar results were seen in Danish and Korean studies examining large cohorts of obese patients $[13,14]$. The classical two-hit hypothesis from which the current complex "multiparallel" theory has been developed to understand the pathogenesis of NASH relies on an initial hit of hepatic steatosis followed by a second hit, which involved IR leading to the release of proinflammatory cytokines [15]. The new multiparallel theory maintains the importance of IR, but includes other pathways such as autophagy and ER stress.

IR in NASH leads to increased levels of tumornecrosis-factor- $\alpha$ (TNF- $\alpha$ ), IL- 6 , and leptin as well as decreased adiponectin [16]. Increased levels of TNF- $\alpha$ leads to activation of NF-KB and allows for hepatic carcinogenesis when exposed to the carcinogen diethylnitrosamine (DEN) [17]. Elimination of expression of IL- 6 as well as the TNF- $\alpha$ receptor reduced $\mathrm{HCC}$ in DEN-treated obese mice $[18,19]$.

IL- 6 and TNF- $\alpha$ also have been shown to increase hepatic production of C-reactive protein (CRP) and increased IL-6 specifically has been shown to be mainly secreted by hepatic stellate cells responsible for hepatic fibrosis [20]. In the setting of obesity, IL- 6 levels are elevated and weight loss reduces IL-6 and TNF- $\alpha$ levels [21]. High levels of TNF- $\alpha$ and IL- 6 found in IR promotes inflammation and fibrosis may both contribute to NASH severity as well as hepatic carcinogenesis.

Decreased adiponectin seen in IR and high BMI has been consistently demonstrated in the setting of NASH and has also been shown to play a role in hepatic fibrosis [22]. Hypoadiponectinemia accelerates hepatic tumor formation in mice [23]. Adiponectin has also been shown to be important in antiangiogenesis and apoptosis, showing the role of low adiponectin in tumor formation [24].

Conversely, the 167-amino acid protein leptin is elevated in the setting of obesity [25]. Leptin has been shown to be an mediator of hepatic fibrosis as well as a contributor to elevated TNF- $\alpha$ levels [26, 27].

IR with subsequent hyperinsulinemia has also been shown to upregulate the production of insulin-like growth factor (IGF1) and insulin receptor substrate-1 (IRS-1). IGF-1 is a peptide hormone that stimulates growth via cellular proliferation and inhibition of hepatic apoptosis [28]. IGF-1 has been shown to significantly activate mitogen-activate protein kinase (MAPK) as well as increased expression of c-fos and c-jun proto-oncogenes in vitro, which is thought to contribute to hepatic carcinogenesis $[29,30]$.

IRS-1 mediates cytokine signaling pathways and has been shown to be upregulated in the setting of HCC [31, 32]. IRS-1 activation leads to MAPK expression and subsequently, $\mathrm{Wnt} / \beta$-catenin signaling cascades that have been shown to occur frequently in HCC from viral hepatitis infections, ethanol exposure, or NASH $[33,34]$.

The above data conclude that both of insulinmediated factors are involved in hepatocarcinogenesis in cirrhotic and non-cirrhotic NASH patients.

Challenged by the question if obesity could be an independent risk factor for HCC, we aimed to estimate the risk of $\mathrm{HCC}$ in obese patients from South-Eastern Romania, diagnosed with NAFLD, without clinical or imagistic features of liver cirrhosis, in order to evaluate if $\mathrm{HCC}$ can develop in fatty liver in the absence of cirrhosis.

\section{Material and method}

We prospectively studied 214 obese patients with NAFLD diagnosed in Gastroenterology, Oncology and Nutrition Departments of Constanta County Hospital, over a period of 5 years, July 2007June 2012.

Patients with regular/daily alcohol consumption or diagnosed with liver viral infections were excluded.

We evaluated all patients using abdominal ultrasound (US) and serum alpha-fetoprotein every 6 
months, in order to detect the HCC occurrence.

Patients were also explored biologically, regarding the lipid and glucose profile. The total cholesterol, LDL-cholesterol, HDL-cholesterol, triglycerides, the serum glucose and the $\mathrm{HbA}$ were explored in every diagnosed patient and correlations with HCC occurrence were done.

The diagnostic value of AFP was $200 \mathrm{ng} / \mathrm{ml}$, but smaller values in association with focal lesion discovered in US were considered criteria for $\mathrm{HHC}$ diagnosis also. AFP is raised in $~ 75 \%$ of $\mathrm{HCC}$ cases. Its level is reverse proportional with the prognosis. A value of AFP over $400 \mathrm{ng} / \mathrm{ml}$ is predictive for $\mathrm{HCC}$, in $95 \%$ of cases.

The diagnosis of liver cirrhosis and hepatocellular carcinoma were done using US, and confirmed by CT scan or MRI.

Abdominal US was done using Logiq700 and ALOKA PROSOUND equipment.

NAFLD was classified using the FIBROSCAN, a device used to determine the value of liver stiffness (EchoSens, Paris, France). The liver stiffness was used to stage the degree of liver fibrosis.

\section{Statistical analyzes}

Kaplan-Meier analysis estimated the cumulative incidence of HCC.

Univariate and multivariate Cox regression analysis were used to assess associations between $\mathrm{HCC}$ and obesity.

\section{Results}

The median follow-up was 4.3 years.

The mean age of HCC diagnosed patients was $65 \pm 11.34$ years (Table I). We observed a decrease in the mean age with every study interval. It was a statistic significance between the mean age obtain in the first interval and the mean age obtain in the last one. ( $p=0,0321$, respectively, $p=0,0487)$ and the tendency of age was to decreases with every studied interval (table no I).
Table I - The mean age of patients with obesity and HCC.

\begin{tabular}{|c|c|}
\hline Interval of study & Age \pm DS \\
\hline ian. 2007 - dec .2007 & $68.20 \pm 10.99$ \\
\hline ian. 2008 - dec. 2008 & $66.20 \pm 12.01$ \\
\hline ian. 2009 - dec. 2009 & $65.28 \pm 8.88$ \\
\hline ian. 2010 - dec. 2010 & $64.66 \pm 10.32$ \\
\hline ian. 2011 - dec. 2011 & $64.03 \pm 8.23$ \\
\hline
\end{tabular}

Comparing our study group with a control group of 28 patients matched by age and sex, admitted in the hospital for the neoplastic pathology but without obesity, we observed that the mean age of diagnosis was significantly bigger than that of obese patients ( $\mathrm{p}=0.0401,95 \%$ CI 25.55-69.91)

Multivariate regression analysis revealed also that older age $(p=0.04)$ was independent variable associated with development of HCC in patients with/without NAFLD-cirrhosis.

Regarding the lipid and glucose profile, our patients revealed the following data (Figure 1):

- 179 of 214 pts. (83.64\%) had lipid and glucose metabolism disturbances;

- 133of 214 pts. (62.14) had only lipid profile disturbances;

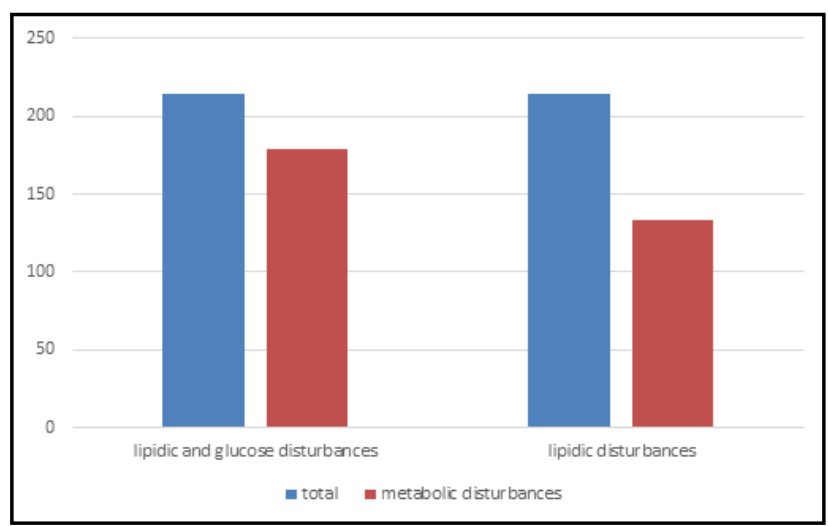

Figure 1 - The distribution of patients according to presence of metabolic disturbances

According to the presence of cirrhosis, abdominal US split our patients in 2 groups (Figure 2):

- 118 patients with cirrhosis

- 96 patients without sings of liver cirrhosis. 


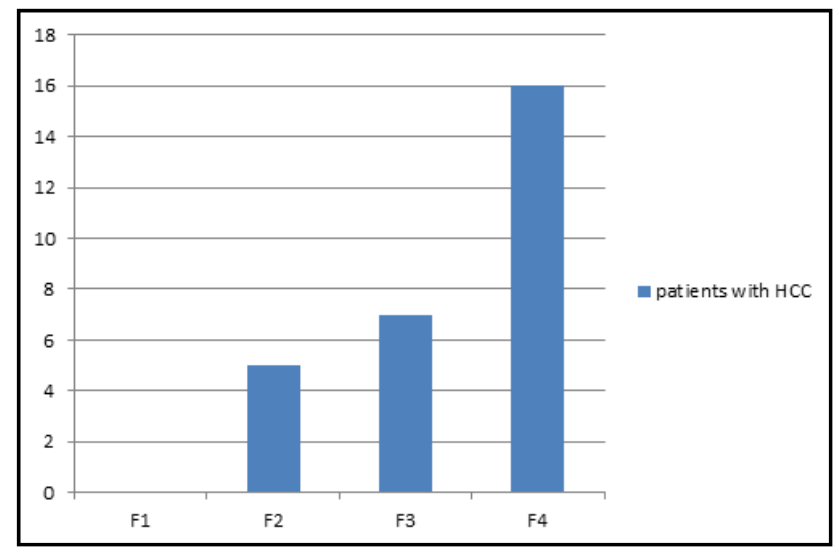

Figure 2 - The classification of patients according with the stage of fibrosis using FIBROSCAN

The diagnosis of NAFLD, and mainly of $\mathrm{NASH}$, requires a liver biopsy [35], which is an invasive procedure and is not without potential risks or complications [36, 37]. fibrosis.

We used FIBROSCAN in order to stage the

According to the value measured in $\mathrm{kPa}$, our patients were classified as follows:

- 16 patients with F4;

- 7 patients with F3;

- 5 patients with F2;

- 0 patients with F1.

During the study period, 16 from 118 cirrhotic NAFLD patients (13.5\%) and 12 from 96 noncirrhotic NAFLD patients (12.5\%) developed HCC $(\mathrm{p}=0.07, \mathrm{~ns})($ Figure 3$)$.

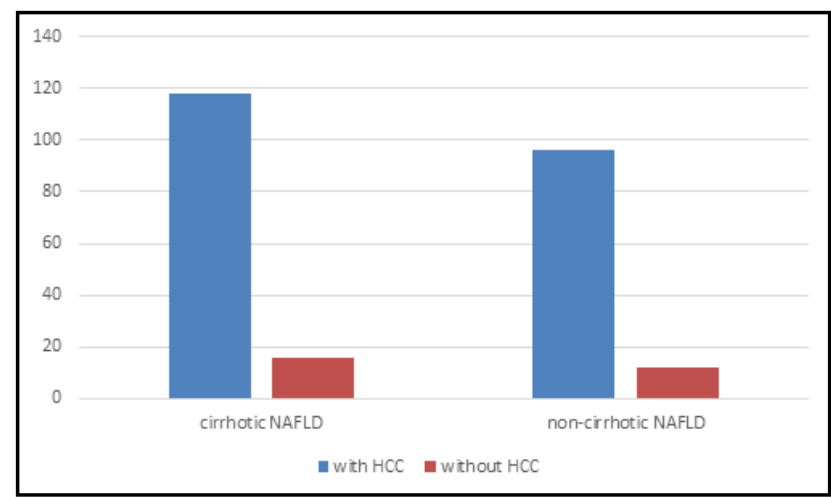

Figure 3 - The distribution of patients according to presence of cirrhosis.
The Kaplan Meyer analyze showed that the cumulative incidence of HCC was found to be $2.9 \%$ in obese patients with NAFLD-cirrhosis, compared with $2.2 \%$ in obese patients without cirrhosis $(\mathrm{p}=$ $0.09, \mathrm{~ns})$.

\section{Discussions}

Obesity has become a universal public health problem with increasing prevalence in both adults and children especially in developed countries, but even in developing ones. Epidemiological studies revealed a strong link between obesity and development and progression of various types of cancers. The connection between obesity and liver cancer is particularly strong and obesity often results in liver diseases such as nonalcoholic fatty liver disease (NAFLD) and the more severe non-alcoholic steatohepatitis (NASH). NASH is characterized by fatty liver inflammation and is believed to cause fibrosis and cirrhosis. The latter is a known liver cancer risk factor. In fact due to its much higher prevalence obesity may be a more substantial contributor to overall hepatocellular carcinoma burden than infection with hepatitis viruses [38]. Our study results came to confirm data from literature, regarding the involvement of obesity in HCC occurrence in the various NAFLD findings, but not with the compulsoriness of cirrhosis presence. The cumulative incidence of HCC was found to be $2.9 \%$ in obese patients with NAFLD-cirrhosis, compared with $2.2 \%$ in obese patients without cirrhosis $(\mathrm{P}=$ $0.09, \mathrm{~ns})$.

\section{Conclusions}

Obesity seems to be an independent risk factor for HCC occurrence, regardless the presence of mild or advanced liver fibrosis in NAFLD patients. 


\section{References}

1. Chen, C.L., Yang, H.I. \& Yang, W.S., et al. (2008). Metabolic factors and risk of hepatocellular carcinoma by chronic hepatitis B/C infection: a follow-up study in Taiwan. Gastroenterology. 135(1), 111-121.

2. Starley, B.Q., Calcagno, C.J. \& Harrison, S.A., (2010). Nonalcoholic fatty liver disease and hepatocellular carcinoma: a weighty connection. Hepatology. 51(5), 1820-32.

3. Caldwell, S.H., Crespo, D.M., Kang, H.S. \& AlOsaimi, A.M., (2004). Obesity and hepatocellular carcinoma. Gastroenterology. 127 (5 Suppl 1), S97-103.

4. Ascha, M.S., Hanouneh, I.A., Lopez, R., Tamimi, T.A., Feldstein, A.F. \& Zein, N.N., (2010). The incidence and risk factors of hepatocellular carcinoma in patients with nonalcoholic steatohepatitis. Hepatology. 51(6), 1972-8.

5. Inoue, M., Iwasaki, M., Otani, T., Sasazuki, S., Noda, M. \& Tsugane, S. (2006). Diabetes mellitus and the risk of cancer: results from a large-scale population-based cohort study in Japan. Arch Intern Med. 166, 1871-7.

6. Inoue, M., Noda, M., Kurahashi, N., Iwasaki, M., Sasazuki, S., Iso, H. \& Tsugane, S., (2009 Jun). Japan Public Health Center-based Prospective Study Group. Impact of metabolic factors on subsequent cancer risk: results from a large-scale population-based cohort study in Japan. Eur $J$ Cancer Prev. 18(3), 240-7.

7. Oh, S.W., Yoon, Y.S. \& Shin, S.A., (2005). Effects of excess weight on cancer incidences depending on cancer sites and histologic findings among men: Korea National Health Insurance Corporation Study. J ClinOncol. 23, 4742-54.

8. Gupta, K., Krishnaswamy, G., Karnad, A. \& Peiris, A.N. (2002). Insulin: a novel factor in carcinogenesis. Am J Med Sci. 323, 140-5.

9. El-Serag, H.B., Tran, T. \& Everhart, J.E. (2004). Diabetes increases the risk of chronic liver disease and hepatocellular carcinoma. Gastroenterology. $126,460-8$.
10. Caldwell, S.H., Crespo, D.M., Kang, H.S. \& AlOsaimi, A.M. (2004). Obesity and hepatocellular carcinoma. Gastroenterology. 127, S97-103.

11. Marrero, J.A., Fontana, R.J., Fu, S., Conjeevaram, H.S., Su, G.L. \& Lok, A.S. (2005). Alcohol, tobacco and obesity are synergistic risk factors for hepatocellular carcinoma. J Hepatol. 42, 218-24.

12. Calle, E.E., Rodriguez, C., Walker-Thurmond, K. \& Thun, M.J. (2003). Overweight, obesity, and mortality from cancer in a prospectively studied cohort of U.S. adults. $N$ Engl J Med. 348(17), 1625-1638.

13. Møller, H., Mellemgaard, A., Lindvig, K. \& Olsen, J.H. (1994). Obesity and cancer risk: a Danish record-linkage study. Eur $J$ Cancer. 30A(3), 344-350.

14. Oh, S.W., Yoon, Y.S. \& Shin, S.A. (2005). Effects of excess weight on cancer incidences depending on cancer sites and histologic findings among men: Korea National Health Insurance Corporation Study. J Clin Oncol. 23(21), 47424754.

15. Tilg, H. \& Moschen, A.R. (2010). Evolution of inflammation in nonalcoholic fatty liver disease: the multiple parallel hits hypothesis. Hepatology. 52(5), 1836-1846.

16. Hashimoto, E. \& Tokushige, K. (2012). Hepatocellular carcinoma in nonalcoholic steatohepatitis: Growing evidence of an epidemic? Hepatol Res. 42(1), 1-14.

17. Wang, Y., Ausman, L.M., Greenberg, A.S., Russell, R.M. \& Wang, X.D. (2009). Nonalcoholic steatohepatitis induced by a highfat diet promotes diethylnitrosamine-initiated early hepatocarcinogenesis in rats. Int J Cancer. 124(3), 540-546.

18. Park, E.J., Lee, J.H. \& Yu, G.Y. et al (2010). Dietary and genetic obesity promote liver inflammation and tumorigenesis by enhancing IL-6 and TNF expression. Cell. 140(2), 197-208.

19. Roderburg, C. \& Trautwein, C. (2010). Obesity and liver cancer: a key role for interleukin- 6 and signal transducer and activator of transcription 3? Hepatology. 51(5), 1850-1852.

20. Sun, B. \& Karin, M. (2011). Obesity, inflammation, and liver cancer. $J$ Hepatol; In 
press.

21. Bougoulia, M., Triantos, A. \& Koliakos, G. (2006). Effect of weight loss with or without orlistat treatment on adipocytokines, inflammation, and oxidative markers in obese women. Hormones (Athens). 5(4), 259-269.

22. Gannagé-Yared, M.H., Khalife, S., Semaan, M., Fares, F., Jambart, S. \& Halaby, G. (2006). Serum adiponectin and leptin levels in relation to the metabolic syndrome, androgenic profile and somatotropic axis in healthy non-diabetic elderly men. Eur J Endocrinol. 155(1), 167-176.

23. Kamada, Y., Matsumoto, H. \& Tamura, S. et al. (2007). Hypoadiponectinemia accelerates hepatic tumor formation in a nonalcoholic steatohepatitis mouse model. J Hepatol. 47(4), 556-564.

24. Bråkenhielm, E., Veitonmäki, N. \& Cao, R. et al. (2004). Adiponectin-induced antiangiogenesis and antitumor activity involve caspase-mediated endothelial cell apoptosis. ProcNatlAcadSci USA. 101(8), 2476-2481.

25. Tsochatzis, E.A., Papatheodoridis, G.V. \& Archimandritis, A.J. (2009). Adipokines in nonalcoholic steatohepatitis: from pathogenesis to implications in diagnosis and therapy. Mediators Inflamm. 8(3), 16-27.

26. Ikejima, K., Honda, H. \& Yoshikawa, M. et al. (2001). Leptin augments inflammatory and profibrogenic responses in the murine liver induced by hepatotoxic chemicals. Hepatology. 34(2), 288-297.

27. Marra, F. (2002). Leptin and liver fibrosis: a matter of fat. Gastroenterology. 122, 1399-1410.

28. Ish-Shalom, D., Christoffersen, C.T. \& Vorwerk, P. et al. (1997). Mitogenic properties of insulin and insulin analogues mediated by the insulin receptor. Diabetologia. 40 (Suppl 2), S25-S31.

29. Buzzelli, G., Dattolo, P., Pinzani, M., Brocchi, A., Romano, S. \& Gentilini, P. (1993). Circulating growth hormone and insulin-like growth factor-I in nonalcoholic liver cirrhosis with or without superimposed hepatocarcinoma: evidence of an altered circadian rhythm. Am J Gastroenterol. 88(10), 1744-1748.

30. Price, J.A., Kovach, S.J. \& Johnson, T. et al. (2002). Insulin-like growth factor I is a comitogen for hepatocyte growth factor in a rat model of hepatocellular carcinoma. Hepatology. 36(5), 1089-1097.

31. Tanaka, S., Mohr, L., Schmidt, E.V., Sugimachi, K. \& Wands, J.R. (1997). Biological effects of human insulin receptor substrate-1 overexpression in hepatocytes. Hepatology. 26(3), 598-604.

32. Aleem, E., Nehrbass, D., Klimek, F., Mayer, D. \& Bannasch, P. (2011). Upregulation of the insulin receptor and type I insulin-like growth factor receptor are early events in hepatocarcinogenesis. ToxicolPathol. 39(3), 524-543.

33. Longato, L., de la Monte, S. \& Kuzushita, N. et al. (2009). Overexpression of insulin receptor substrate-1 and hepatitis $\mathrm{Bx}$ genes causes premalignant alterations in the liver. Hepatology. 49(6), 1935-1943.

34. Longato, L., de la Monte, S., Califano, S. \& Wands, J.R. (2008). Synergistic premalignant effects of chronic ethanol exposure and insulin receptor substrate-1 overexpression in liver. Hepatol Res. 38(9), 940-953.

35. Adams, L.A. \& Feldstein, A.E. (2010). Nonalcoholic steatohepatitis: risk factors and diagnosis. Expert Rev GastroenterolHepatol. 4, 623-35.

36. Cadranel, J.F., Rufat, P. \& Degos, F. (2000). Practices of liver biopsy in France: results of a prospective nationwide survey. For the Group of Epidemiology of the French Association for the Study of the Liver (AFEF). Hepatology; 32, 477-81.

37. Festi, D., Schiumerini, R., Marzi, L., Di Biase, A. R., Mandolesi, D., Montrone, L., Scaioli, E., Bonato, G., Marchesini-Reggiani, G. \& Colecchia, A. (2013). Review Article: The Diagnosis of Non-alcoholic Fatty Liver Disease Availability and Accuracy of Non-invasive Methods. Aliment PharmacolTher. 37(4), 392400.

38. Sun, B. \& Karin, M. (2012 Mar). Obesity, inflammation, and liver cancer. J Hepatol. 56(3), 704-13. DOI: 10.1016/j.jhep.2011.09.020. 